\title{
Paradoxical akathisia caused by clonazepam, clorazepate and lorazepam in patients with traumatic encephalopathy and seizure disorders: a subtype of benzodiazepine-induced disinhibition?
}

\author{
A.B. Joseph ${ }^{1}$ and B.A. Wroblewski \\ 'McLean Hospital, Belmont, Center for Neurobehavioral Rehabilitation, Waltham, and \\ Harvard Medical School, Boston, MA, and ${ }^{2}$ Greenery Rehabilitation Center, Boston, \\ Department of Rehabilitation Medicine, Tufts University School of Medicine, Boston and \\ Massachusetts College of Pharmacy and Allied Health Sciences, Boston, MA, USA
}

Correspondence to: A.B. Joseph, Center for Neurobehavioral Rehabilitation, 775 Trapelo Road, Waltham, MA 02154, USA

\begin{abstract}
Akathisia is frequently reported to be caused by neuroleptic drugs and sometimes by certain other agents such as fluoxetine. Benzodiazepines are a common treatment. The principal mechanism of akathisia is thought to be neurochemical, probably dopaminergic with serotonin also playing an important role. It is not usually thought to be related to benzodiazepine-caused disinhibition. Four episodes of atypical or paradoxical benzodiazepine-induced akathisia in three patients are reported and analyzed. All four episodes of akathisia were atypical because they were caused by clonazepam, clorazepate, or lorazepam. In one patient neither thiothixene nor lorazepam caused akathisia, but clonazepam and clorazepate did. In another patient both lorazepam and fluoxetine caused akathisia. It is also noted that all three patients had a history of traumatic brain injury and seizure disorder. The data support the hypothesis that atypical benzodiazepine-induced akathisia exists. Its mechanism may be different from neuroleptic-induced akathisia, but may still involve serotonergic systems or the forced normalization phenomenon. The similarity of these cases to reports of benzodiazepine-induced disinhibition raises the possibility that in some patients they may be the same entity.
\end{abstract}

Keywords: Akathisia - Clonazepam - Clorazepate - Disinhibition - Forced normalization - Lorazepam

\section{INTRODUCTION}

Akathisia, a combination of motor restlessness and psychic anxiety, is often reported after treatment with high potency neuroleptic drugs such as haloperidol and thiothixene but also has other causes. Treatments are usually thought to include such drug classes as benzodiazepines, beta-blockers, and anticholinergics. We report four episodes of akathisia caused by clonazepam, clorazepate and lorazepam, benzodiazepine drugs, in three patients with traumatic encephalopathy and seizure disorders.

\section{CASE 1: AKATHISIA CAUSED BY CLONAZEPAM AND CLORAZEPATE, BUT NOT LORAZEPAM OR THIOTHIXENE}

A 40 year old right-handed Black woman presented for treatment of various behavioral syndromes including: atypical psychosis, marked by paranoia, visual and audi- tory hallucinations; Cotard's syndrome; palinopsia (visual perserveration); palinaesthesia (tactile perseveration); autoscopy; Fregoli and intermetamorphosis syndromes (delusional misidentification syndromes); and sexual seizures (of simple partial seizure type). Her past medical history was remarkable for traumatic head injury and loss of consciousness 20 years previously and a subsequent seizure disorder. Her behavioral and psychotic symptoms started 9 years after the trauma. Her family history was positive for a grandmother with Alzheimer's disease and paranoia, and a father with chronic psychosis. She had no history of movement disorder.

Brain electrical activity mapping (BEAM) revealed left anterior temporal abnormalities consistent with a seizure disorder. Neuropsychological testing revealed significant impairment in short-term memory and visuo-spatial tasks consistent with an organic etiology. 
Treatment was initiated with $5 \mathrm{mg} /$ day of thiothixene and $1.5 \mathrm{mg} /$ day of lorazepam. The patient's psychotic symptoms responded well to this approach but she reported sedation and mild cognitive impairment from the thiothixene. As treatment progressed, she finally felt comfortable with thiothixene reduced to a dose of $2 \mathrm{mg} /$ day, but her symptoms persisted. While continuing on thiothixene lorazepam was gradually increased, with good symptom resolution, to $6 \mathrm{mg} /$ day. At this point sedation continued to be difficult for her, and approximately 7 months after starting thiothixene, clonazepam at $6 \mathrm{mg} /$ day was substituted for lorazepam. Within several days of starting clonazepam, the patient reported an extremely unpleasant feeling of subjective inner anxiety and restlessness coupled with driven motor behavior and relentless pacing; in short, akathisia. Decreasing clonazepam to $2 \mathrm{mg} /$ day did not improve these side effects, and it was discontinued within a week, after which they rapidly resolved. Lorazepam was restarted at $1.5 \mathrm{mg} /$ day, and subsequently gradually increased to $9 \mathrm{mg} /$ day. At that dose she reported almost complete symptom control, once again without any akathisia.

Five months later, at a daily dose of $8 \mathrm{mg}$ of lorazepam, paranoia and visual hallucinations recurred. A trial of clorazepate was initiated at $3.75 \mathrm{mg} /$ day to give better control of her psychotic symptoms. Sedation became a problem and lorazepam was decreased and clorazepate increased. Within a month her psychosis had improved on $15 \mathrm{mg} /$ day clorazepate and $6 \mathrm{mg}$ /day lorazepam. Clorazepate was then increased to $22.5 \mathrm{mg} /$ day. Within several days she developed insomnia and complained of being woken every $2 \mathrm{~h}$ by extreme anxiety. She also reported severe inner restlessness. Both these symptoms resolved if she self-medicated with additional lorazepam. She also suffered from akathisia and increased visual hallucinations and paranoia. She reported that the clorazepate activated and agitated her. It was discontinued and the akathisia resolved within a week. At a dose of $8 \mathrm{mg}$ /day lorazepam she returned to her previously described baseline with acceptable control of psychotic symptoms.

\section{CASE 2: AKATHISIA CAUSED BY CLORAZEPATE}

A 34 year old ambidextrous man had suffered a head injury with traumatic loss of consciousness in 1980. In 1984 he developed grand mal, complex partial, and absence seizures. As a child he had attention deficit hyperactivity disorder. He had no history of movement disorder. Ongoing target behaviors included temper tantrums, verbally threatening and abusive behavior, and physical intimidation of others. His past history was otherwise unremarkable.

His seizures had been difficult to control on tegretol and he was started on clorazepate $7.5 \mathrm{mg}$ b.i.d. as an adjunctive anticonvulsant. He had no known prior history of exposure to dopamine-blocking agents.

During the following 3 weeks he developed severe akathisia marked by motor restlessness, a need to pace endlessly, and anxiety. He became very irritable, explosive, and suicidally depressed, finally requiring admission to a locked psychiatric unit.

He described the effect of clorazepate as making him feel "wired", and causing depression and the symptoms noted above. These almost completely resolved as clorazepate was tapered to $1.875 \mathrm{mg}$ every other day. At this point the patient was lost to follow-up.

\section{CASE 3: AKATHISIA CAUSED BY LORAZEPAM AND FLUOXETINE}

A 34 year old right-handed woman suffered a traumatic brain injury and loss of consciousness at age 8 when she had been hit by a truck. She also suffered from chronic psychosis, believing that other people lived within her; post-traumatic stress disorder, after having been sexually abused as a child; a chronic seizure disorder marked by simple and complex partial seizure types; and major depressive disorder. In the past, many years before assessment by one of us (A.B.J.), she had abused alcohol, cocaine, LSD, marijuana and amphetamines. She had no known history of movement disorder or exposure to dopamine-blocking agents.

At presentation her target symptoms included: various neurovegetative symptoms of depression; racing thoughts; auditory command hallucinations to "smash my car"; frequent intense déjà $v u$; and forced thinking. She was prescribed lorazepam $0.5 \mathrm{mg}$ b.i.d. which she took for 3 days and then discontinued because of increased anxiety, a feeling of restlessness and a need to pace and move constantly. She described the effect as follows: "(I) had to run ... always moving ... always doing something ... I couldn't sit (still)." She had to keep moving her legs to relieve the tension. Her epileptic symptoms were not improved. Within 2 days of discontinuing lorazepam the side effects had resolved.

One week later she rechallenged herself with the same dose of lorazepam for another 2 days and the same side effects recurred, again resolving 2 days after discontinuing lorazepam for the second time.

A diagnosis of akathisia secondary to lorazepam was made and the patient was started on fluoxetine $20 \mathrm{mg} /$ day to treat her continuing depression. At follow-up she reported discontinuing fluoxetine after two doses because of side effects. After the first dose she reported becoming homicidally angry, more depressed, having increased racing thoughts, feeling restless, agitated and compelled to pace, as with lorazepam. In her words: "It made me wired 
... I couldn't sit down, I had to be always moving." These side effects lasted 18-24 h after the initial dose of fluoxetine and gradually resolved during that time. The same events repeated after the second dose and the patient discontinued the medication.

A diagnosis of akathisia secondary to fluoxetine was made.

\section{DISCUSSION}

The authors were unable to find other reports in the literature of clonazepam-, clorazepate-, or lorazepam-induced akathisia.

It is noteworthy that the first patient did not experience cognitive or motor akathisia prior to or after the week of clonazepam even when she was on a potent neuroleptic. Patient 2 clearly suffered from new onset akathisia when clorazepate was added to his treatment regimen, and this essentially resolved once the dose was negligible. Patient 3 rechallenged herself and consistently had akathisia secondary to low doses of both lorazepam and fluoxetine.

The explanation for these episodes of akathisia is not at all clear, but two possibilities are that they were due to clonazepam's serotonergic or anticonvulsant properties or to clorazepate's and lorazepam's anticonvulsant activity (Cohen and Rosenbaum, 1987; Fuji et al., 1987). Akathisia, often thought to depend on dopaminergic activity, has also been related to serotonergic inhibition of dopaminergic activity. This mechanism has been offered as an explanation of fluoxetine- and tricyclic antidepressantinduced akathisia (Lipinski et al., 1989). It is clear, then, that theoretically clonazepam's serotonergic effect could be expected to cause akathisia. Clinically, however, like other benzodiazepines, it is usually thought of as a treatment (Kutcher et al., 1989).

Unusually, in Patient 1, clonazepam induced akathisia. This case thus supports one theory of the causation of akathisia, but at the same time contradicts another theory of its treatment. It is also possible that this episode of akathisia was secondary to the anticonvulsant rather than the serotonergic properties of clonazepam. With this possibility in mind it is noteworthy that Patient 1 developed akathisia again when given clorazepate, a potent anticonvulsant, as did Patient 2, and that Patient 3 developed akathisia from lorazepam, also an anticonvulsant. In the phenomenon of forced normalization, just such a worsening of psychiatric symptoms is said to happen as a patient's seizures decrease and their EEG improves or "normalizes" (Wolf, 1986).

In the patients reported here there is little direct evidence that such a phenomenon occurred. Nevertheless, all had suffered traumatic brain injuries in the past which supported ongoing poorly controlled seizure disorders. That they developed akathisia when given potent anticonvul- sants suggests that forced normalization must at least be considered as a possible mechanism.

Forced normalization has also been reported to rarely cause psychotic motor agitation in patients taking carbamazepine or valproic acid (Pakalnis et al., 1987). In children specifically, it has been suggested that it is the way in which benzodiazepines can cause agitated motor behavior (Trimble and Cull, 1989). Clearly forced normalization could in theory have caused the psychomotor agitation our patients suffered. This possibility raises a significant question. The psychomotor agitation reported herein was of a specific type: akathisia. If benzodiazepines caused akathisia in our patients, is it possible that at least a subgroup of benzodiazepine-induced "disinhibition" is in fact paradoxical akathisia instead?

In summary, these cases represent examples of "paradoxical" akathisia for two reasons. The first apparent paradox is that Patient 1's initial episode of akathisia was caused by a benzodiazepine and was unrelated to the potent neuroleptic she was taking, implying an atypical mechanism. The second is that in the three patients described here, benzodiazepines, a common treatment of akathisia, actually caused it instead.

It may be that paradoxical akathisia is due to a novel mechanism distinct from that which is involved in the neuroleptic-induced variety. It may also be that paradoxical akathisia is a subtype of benzodiazepine-induced disinhibition. Verifying the existence of paradoxical akathisia, establishing its mechanism, and exploring its relationship to disinhibition will need to await the description and investigation of other cases.

\section{REFERENCES}

Cohen LS and Rosenbaum JF (1987) Clonazepam: new uses and potential problems. Journal of Clinical Psychiatry, 48 (10, Suppl.), 50-55.

Fuji T, Okuno T, Go T, Ochi J, Hattori H, Kataoka K and Mikawa H (1987) Clorazepate therapy for intractable epilepsy. Brain and Development, 9, 288-291.

Kutcher S, Williamson P, MacKenzie S et al. (1989) Successful clonazepam treatment of neuroleptic-induced akathisia in older adolescents and young adults: a double blind, placebocontrolled study. Journal of Clinical Psychopharmacology, 9, 403-406.

Lipinski JF, Mallya G, Zimmerman P and Pope HG (1989) Fluoxetine-induced akathisia: clinical and theoretical implications. Journal of Clinical Psychiatry, 50, 339-342.

Pakalnis A, Drake ME Jr, John K and Kellum JB (1987) Forced normalization. Acute psychosis after seizure control in seven patients. Archives of Neurology, 44, 289-292.

Trimble MR and Cull CA (1989) Antiepileptic drugs, cognitive function, and behavior in children. Cleveland Clinic Journal of Medicine, 56 (Suppl. Pt. 1), 140-149.

Wolf $\mathrm{P}$ (1986) Forced normalization. In: Aspects of Epilepsy and Psychiatry (Eds MR Trimble and TG Bolwig). John Wiley and Sons.

(Received 1 November 1992; accepted as revised 19 September 1993) 


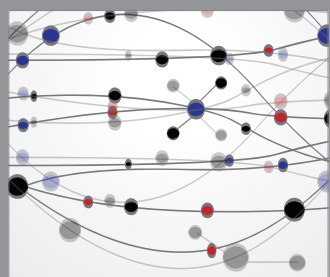

The Scientific World Journal
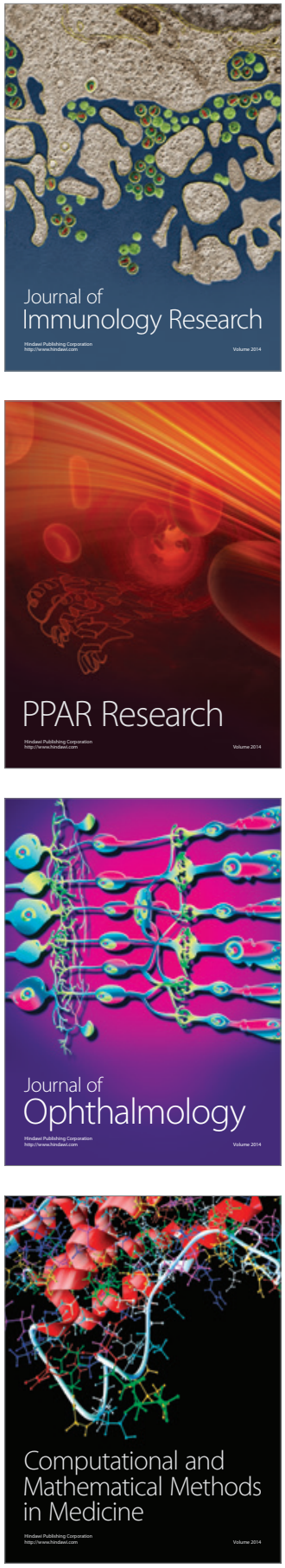

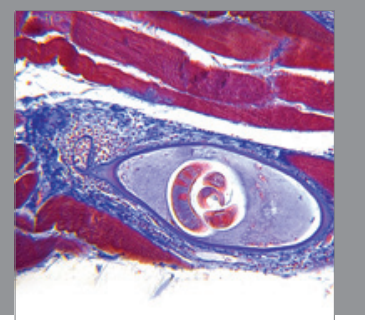

Gastroenterology

Research and Practice
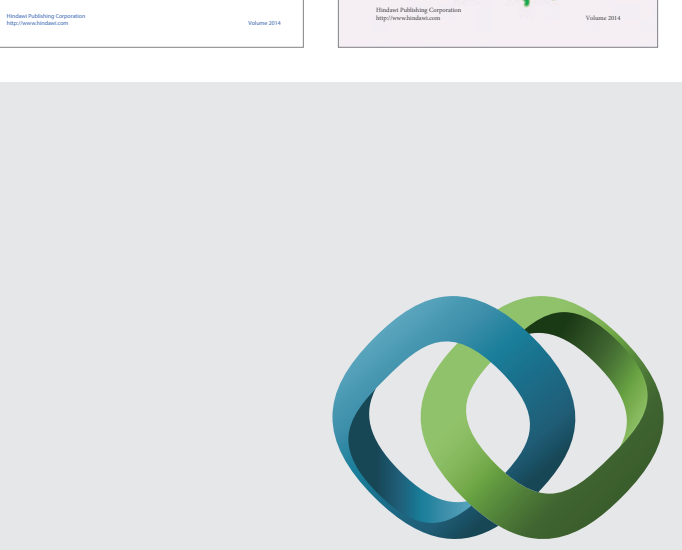

\section{Hindawi}

Submit your manuscripts at

http://www.hindawi.com
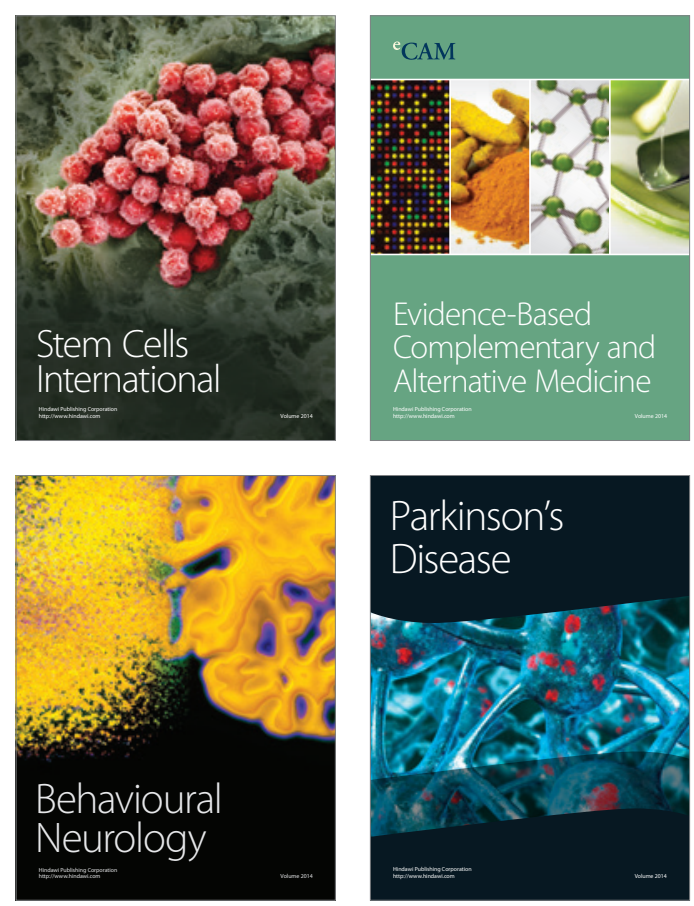

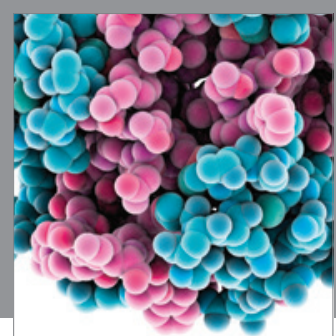

Journal of
Diabetes Research

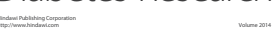

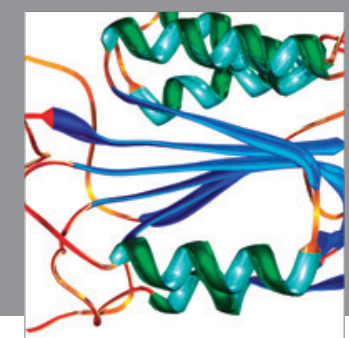

Disease Markers
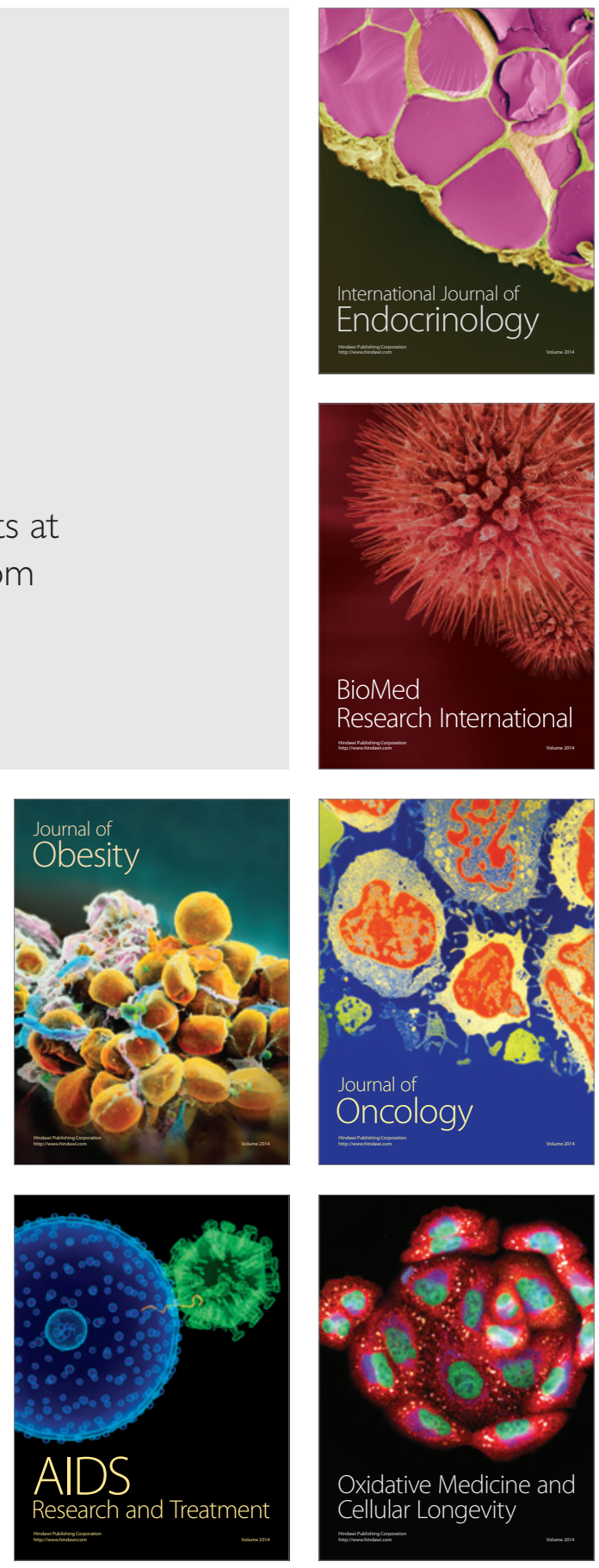